\title{
On an emotional node: modeling sentiment in graphs of action verbs
}

\author{
Petersen, Michael Kai; Hansen, Lars Kai
}

Published in:

2012 International Conference on Audio, Language and Image Processing (ICALIP)

Link to article, DOI:

10.1109/ICALIP.2012.6376631

Publication date:

2012

Link back to DTU Orbit

Citation (APA):

Petersen, M. K., \& Hansen, L. K. (2012). On an emotional node: modeling sentiment in graphs of action verbs. In 2012 International Conference on Audio, Language and Image Processing (ICALIP) https://doi.org/10.1109/ICALIP.2012.6376631

\section{General rights}

Copyright and moral rights for the publications made accessible in the public portal are retained by the authors and/or other copyright owners and it is a condition of accessing publications that users recognise and abide by the legal requirements associated with these rights.

- Users may download and print one copy of any publication from the public portal for the purpose of private study or research.

- You may not further distribute the material or use it for any profit-making activity or commercial gain

- You may freely distribute the URL identifying the publication in the public portal

If you believe that this document breaches copyright please contact us providing details, and we will remove access to the work immediately and investigate your claim 


\title{
ON AN EMOTIONAL NODE: MODELING SENTIMENT IN GRAPHS OF ACTION VERBS
}

\author{
Michael Kai Petersen, Lars Kai Hansen
}

\author{
Cognitive Systems, DTU Informatics, Technical University of Denmark \\ Building 321, DK-2800 Kgs.Lyngby, Denmark \\ $\{$ mkp, lkh\}@imm.dtu.dk
}

\begin{abstract}
Neuroimaging studies have over the past decades established that language is grounded in sensorimotor areas of the brain. Not only action verbs related to face and hand motion but also emotional expressions activate premotor systems in the brain. Hypothesizing that patterns of neural activation might be reflected in the latent semantics of words, we apply hierarchical clustering and network graph analysis to quantify the interaction of emotion and motion related action verbs based on two large-scale text corpora. Comparing the word topologies to neural networks we suggest that the co-activation of associated word forms in the brain resemble the latent semantics of action verbs, which may in turn reflect parameters of force and spatial differentiation underlying action based language.
\end{abstract}

Index Terms - action verbs, graph analysis, LSA

\section{INTRODUCTION}

Computational models in neuroimaging for predicting how objects like 'celery' or 'airplane' are represented in the brain, have successfully been trained by modeling how these concepts as nouns co-occur within multiple contexts retrieved from large-scale text corpora [1]. Likewise verbs can be used to model how we conceptualize not only actions but also feelings. Mounting evidence for a multimodal mapping between motion and words has provided a foundation for the recently proposed theory of action-based language [2], where meaning is defined as action schemas that relate objects in a space constrained by parameters of force and motion. In turn reflecting how mirror neurons in the brain translate spatial positions of entities into sequences of motor programs, that enable us to visually locate, reach out and grasp an object [3]. Lately neuroimaging studies have demonstrated that the sensorimotor system provides a binding not only for word categories related to arm or face motion but also for verbs with an abstract emotional meaning [4].

It has been proposed that neurosemantics might benefit from simulating the correlated activation of word forms in language areas of the brain using combinatorial statistics of word co-occurrences in large-scale text corpora [5], whether based on neural networks [6] or latent semantic analysis LSA
[7]. Cognitive component analysis COCA, defined as an unsupervised learning of features resembling how we perceive the world, suggests that sensory structures can be modeled by reducing dimensionality and treating objects in space and time as linear mixtures [8]. Going beyond our initial COCA analysis of 30 action verbs [9], we here take $3 \times 20$ hand, face and emotion related action verbs used in the above neuroimaging study [4], and hypothesize that the force and spatial parameters which define action-based language might also be reflected in the co-variances of words underlying their latent semantic structure. And to explore to what degree these underlying structures might generalize we apply LSA based on two different large-scale text corpora HAWIK and TASA available online ${ }^{12}$. Next we model verbs as nodes in a forcedirected graph to quantify their interaction based on aspects of network graph theory [10]. And subsequently compare the retrieved semantic structures against the neural correlates of emotion and action verbs based on recent neuroimaging studies [11] [12] [4]. In the following sections we outline methods, results and discuss the topological structures which we propose cognitively relate the meaning of emotion verbs to sensorimotor action schemas.

\section{METHODS}

Initially selecting $3 \times 20$ hand, face and emotion related action verbs used in a recent neuroimaging study [4], which applied fMRI functional magnetic resonance imaging to measure hemodynamic responses in a passive reading task, we submit this subset of action verbs to an LSA analysis [7], in order to define similarities between each pair of verbs based on the HAWIK and TASA text corpora. Applying singular value decomposition SVD new matrices of lower dimensionality are generated in which words that have similar meanings in different contexts are squeezed into a reduced number of rows and columns, corresponding to eigenvectors which capture orthogonal directions in the original matrix. Dimensionality is reduced from millions to a few hundred by keeping only

\footnotetext{
${ }^{1}$ Technical University of Denmark: LSA software \& HAWIK corpus matrices http://dl.dropbox.com/u/5442905/LSA.zip DTU Informatics, 2010

${ }^{2}$ University of Colorado: LSA latent semantic analysis based on TASA general reading corpus http://lsa.colorado.edu CU Boulder, 1998
} 


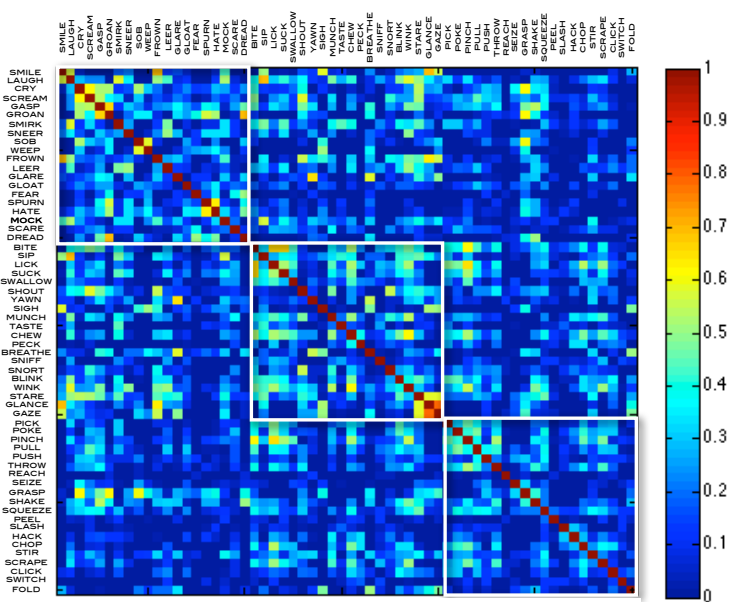

Fig. 1. Adjacency matrix defining the interaction between $3 \times 20$ emotion, face and hand related verbs, weighted by their LSA cosine similarity using the HAWIK corpus, based on 22829 words found in 67380 excerpts of Harvard Classics literature, Wikipedia articles and Reuters news.

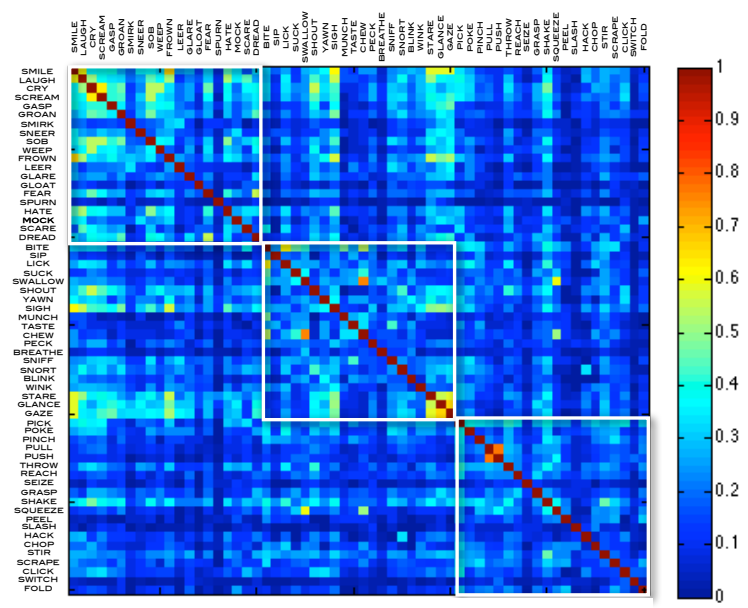

Fig. 2. Adjacency matrix defining the interaction between $3 \times 20$ emotion, face and hand related verbs, weighted by their LSA cosine similarity using the TASA corpus, based on 92409 words found in 37651 texts reflecting the reading material students have been exposed to when entering college. the eigenvectors which correspond to the highest eigenvalues in the original matrix. In order to explore to what degree the latent semantics of action verbs generalize we define cosine similarities based on two different large scale collections of texts HAWIK and TASA. The approach for optimizing and validating the number of eigenvectors have been outlined in our initial study of 30 action verbs [9]. To quantify the connectivity we model the $3 \times 20$ action verbs as nodes using a force directed graph algorithm [13], whereby the links are weighted in proportion to their LSA cosine similarity thresholded at values above 0.20 . Here the strength of node $x_{i}$ is given by its degree and weights of links i.e. the adjacency and weight matrices of nodes $i$ and $j$. We calculate the eigenvector centrality [14], which weights nodes not only based on their degree of connectivity, but similar to Google's PageRank algorithm also takes into consideration whether the links are formed between nodes that are themselves central within the network. That is, for a $n \times n$ matrix $A$ containing pairwise similarity measures the eigenvector centrality $x_{i}$ of node $i$

$x_{i}=\frac{1}{\lambda} \sum_{j=1}^{n} a_{i j} x_{j}$

is defined as the $i$-th entry in the normalized eigenvector belonging to the largest eingenvalue $\lambda$ of $A$

\section{RESULTS}

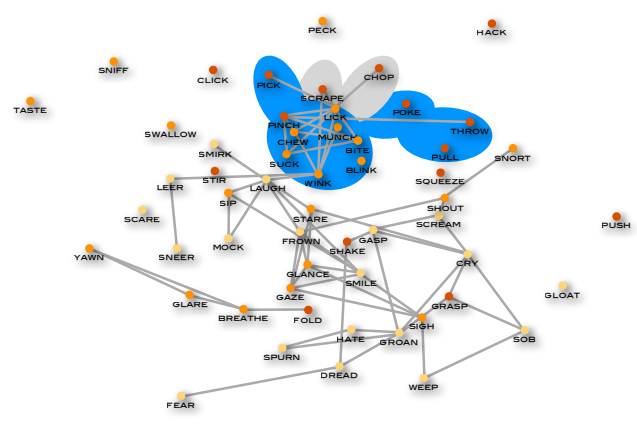

seize

Fig. 3. Force directed graph of nodes and LSA weighted edges based on the HAWIK corpus thresholded above 0.20, which separates clusters of emotion and facial expressions (bottom) from mouth and hand related action verbs (top). Solid lines indicate strong links i.e. LSA similarity above 0.50 . Grey shaded nodes are maximal cliques where the blue shaded verbs remain shared among all subgraphs. Top eigenvector centrality nodes: 'wink', 'bite' and 'laugh'. 


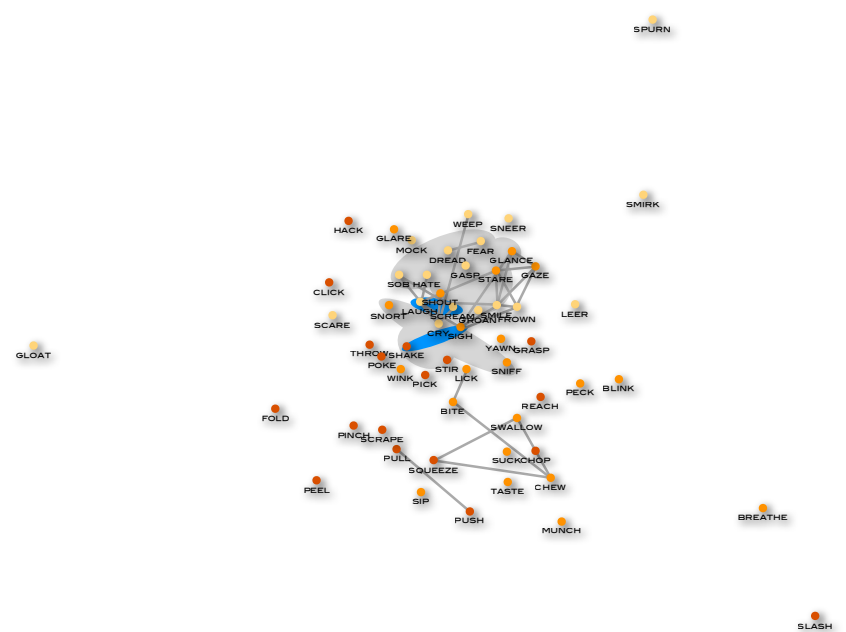

Fig. 4. Force directed graph of nodes and LSA weighted edges based on the TASA corpus thresholded above 0.20, which separates clusters of emotion and facial expressions (top) from mouth and hand related action verbs (bottom). Solid lines indicate strong links i.e. LSA similarity above 0.50. Grey shaded nodes are maximal cliques where the blue shaded verbs remain shared among all subgraphs. Top eigenvector centrality nodes: 'sigh', 'smile' and 'laugh'.

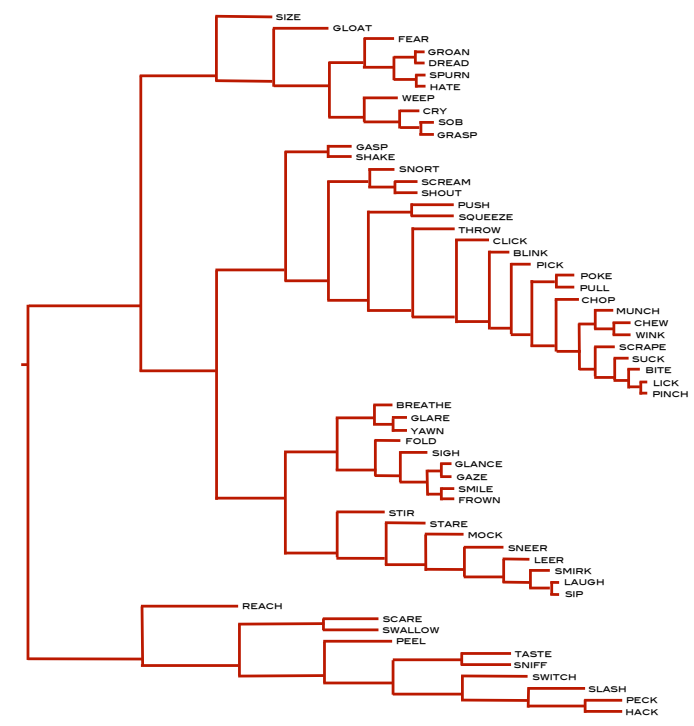

Fig. 5. Hierarchical clustering of $3 \times 20$ emotion, face and hand related action verbs based on HAWIK corpus LSA cosine similarity (top to bottom): aroused expressions, short distance small size mouth and hand gestures ranging from low to high energy, long duration low velocity actions, high velocity short duration gestures.

\section{DISCUSSION}

Modeling the $3 \times 20$ emotion, face and hand related action verbs as nodes within LSA weighted graphs, a number of

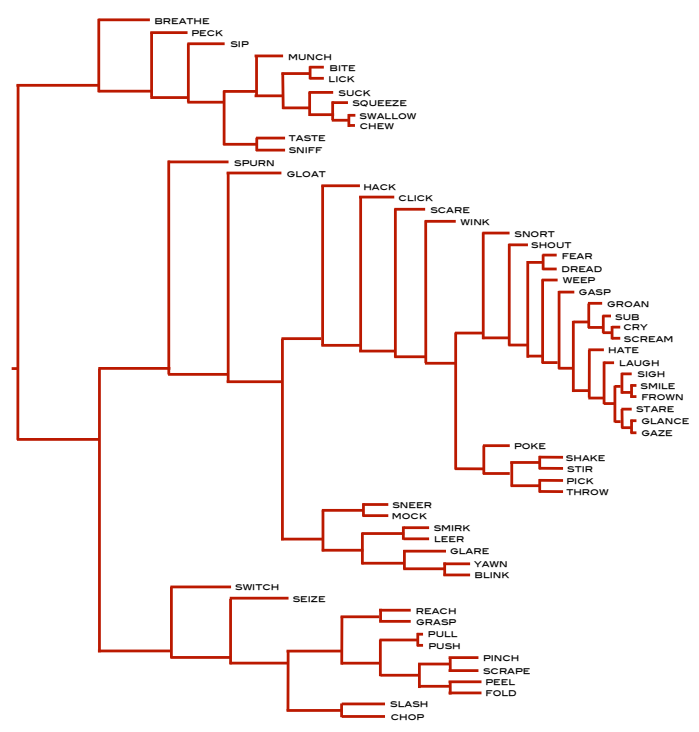

Fig. 6. Hierarchical clustering of $3 \times 20$ action face and hand related action verbs based on TASA corpus LSA cosine similarity (top to bottom): small size closure related mouth actions, aroused emotions and actions of contrasting energy, mostly sustained facial expressions, gestures of high velocity and short duration.

shared characteristics within the HAWIK and TASA seem apparent. In both networks emotional expressions such as 'smile' and 'laugh' are intertwined with clusters of eye motion formed by nodes like 'gaze' and 'glance'. These parts of the networks stand out separate from less strongly linked but fully interconnected maximum cliques of hand and mouth gestures such as 'suck' and 'pinch'. Thereby suggesting modular structures not unlike those identified in the fMRI neuroimaging study including the same action verbs [4], which demonstrated distinct but overlapping patterns characterized by higher activation for emotional verbs in the left prefrontal regions of the brain. Hand action verbs were found to dominate the left lateral premotor and motor regions, whereas face related verbs additionally triggered the supramarginal gyrus in the right hemisphere. Emotional verbs showed a stronger activation in the orbitofronal and dorsolateral prefrontal regions compared to the mouth and hand related verbs. Graph theory emphasizes analysis of connectivity which is likewise relevant for analysis of brain networks, where closely positioned regions have a higher likelihood of being connected, while remote modules are typically linked through high centrality nodes functioning as hubs. The inherent modular structure in brain networks can be estimated by hierarchical clustering, which makes the heavily interlinked subgraphs stand out as they are often only sparsely connected with nodes in other modules [10]. Related to the hierarchical clustering and graph analyses of action verbs, the separation of emotional 
expressions from the hand and mouth related verbs, is in line with neuroanatomical findings of lateralization within the two hemispheres of the brain. Interconnected maximal cliques of verbs such as 'bite' and 'pinch' resemble in functionality the posterior part of Broca's area, involved in both speech processing and hand movement [2]. Eigenvector centrality is used in neuroimaging to identify connectivity patterns in the brain [14]. In the HAWIK graph (Fig.3), the top eigenvector values are found for the nodes 'wink' and 'laugh' which connect the subgraph of emotions and facial expressions with the cluster of mouth and hand related action verbs formed around the node 'bite'. The verb 'laugh' is also among the top eigenvector centrality nodes in the TASA graph (Fig.4), while 'smile' and 'sigh' provide links to clusters of eye motion and the subgraphs of mouth and hand related actions respectively. This division separating close-up mouth and hand gestures from eye and emotional expressions, might reflect differences in attentional processing. Vigilant, alert and sustained attention are primarily right part brain processes related to intensity, whereas selectivity i.e. focused attention is shifted towards the left hemisphere [15]. Finding these topological structures across two large-scale text corpora, we propose that co-activation of associated word forms in the brain might also be reflected in the latent semantics of action verbs. And suggest that cognitive component analysis COCA [8][9], as an unsupervised learning of features reducing dimensionality and treating objects in space and time as linear mixtures, might allow us to retrieve the parameters of force and motion which have been proposed to impose the structure on actionbased language in the sensorimotor networks of the brain [2].

\section{REFERENCES}

[1] Tom M. Mitchell, Svetlana V. Shinkareva, Andrew Carlson, Kai-Min Chang, Vicente L. Malave, Robert A. Mason, and Marcel Adam Just, "Predicting human brain activity asociated with the meanings of nouns," Science, vol. 320, 2008.

[2] Arthur M. Glenberg and Vittorio Gallese, "Actionbased language: A theory of language acquisition, comprehension, and production," Cortex, vol. doi:10.1016/j.cortex.2011.04.01, pp. 1-18, 2011.

[3] Vittorio Gallese and George Lakoff, "The brain's concepts: the role of the sensory-motor system in conceptual knowledge," Cognitive Neuropsychology, vol. 22, no. 3-4, pp. 455-479, 2005.

[4] Rachel Moseley, Francesca Carota, Olaf Hauk, Bettina Mohr, and Friedemann Pulvermüller, "A role for the motor system in binding abstract emotional meaning," Cerebral Cortex, vol. doi:10.1093/cercor/bhr238, pp. 114, 2011.
[5] Friedemann Pulvermüller, "Meaning and the brain: The neurosemantics of referential, interactive, and combinatorial knowledge," Journal of Neurolinguistics, vol. doi:10.1016/ j.jneuroling.2011.03.004, pp. 1-37, 2011.

[6] Friedemann Pulvermüller and Andreas Knoblauch, "Discrete combinatorial circuits emerging in neural networks: a mechanism for rules of grammar in the human brain?," Neural Networks, vol. 22, pp. 161-172, 2009.

[7] Thomas K. Landauer and Susan T. Dumais, "A solution to plato's problem: the latent semantic analysis theory of acquisition, induction and representation of knowledge," Psychological Review, vol. 104, no. 2, pp. 211240, 1997.

[8] Lars Kai Hansen, Peter Ahrendt, and Jan Larsen, "Towards cognitive component analysis," in International and Interdisciplinary Conference on Adaptive Knowledge Representation and Reasoning, 2004.

[9] Michael Kai Petersen and Lars Kai Hansen, "Cognitive semantic networks: emotional verbs throw a tantrum but don't bite," IEEE Explore, vol. in press, 2012.

[10] Ed Bullmore and Olaf Sporns, "Complex brain networks: graph theoretical analysis of structural and functional systems," Nature Neuroscience, vol. 10, pp. 186198, 2009.

[11] Olaf Hauk, Ingrid Johnsrude, and Friedemann Pulvermüller, "Somatotopic representation of action words in human motor and premotor cortex," Neuron, vol. 41, pp. 301-307, 2004.

[12] Javier Rodríguez-Ferreiro, Silvia P. Gennari, Robert Davies, and Fernando Cuetos, "Neural correlates of abstract verb processing," Journal of Cognitive Neuroscience, vol. 23, no. 1, pp. 106-118, 2010.

[13] Thomas M. J. Fruchterman and Edward M. Reingold, "Graph drawing by force-directed placement," Software - Practice and Experience, vol. 21, no. 11, pp. 11291164, 1991.

[14] Gabriele Lohmann, Daniel S. Margulies, Annette Horstmann, Burkhard Pleger, Joeran Lepsien, Dirk Goldhahn, Haiko Schloegl, Michael Stumvoll, Arno Villringer, and Robert Turner, "Eigenvector centrality mapping for analyzing connectivity patterns in fmri data of the human brain," PLoS ONE, vol. 5, no. 4, pp. e10232. doi:10.1371/journal.pone.0010232, 2010.

[15] Ian McGilchrist, The master and his emissary: the divided brain and the making of the western world, Yale University Press, 2009. 